\title{
Changes in Physical Activity across the Lifetime of Current Ultra-Endurance Exercisers
}

\author{
Karen Birkenhead ${ }^{1, *}$, Geoff Lovell ${ }^{2}$, Susan I. Barr ${ }^{3}$, Colin Solomon ${ }^{4}$ \\ ${ }^{1}$ School of Health and Sports Sciences, University of the Sunshine Coast, Maroochydore, 4558, Australia \\ ${ }^{2}$ School of Social Sciences, University of the Sunshine Coast, Maroochydore, 4558, Australia \\ ${ }^{3}$ Food, Nutrition and Health, University of British Columbia, Vancouver, V6T 1Z4, Canada \\ ${ }^{4}$ School of Health and Sports Sciences, University of the Sunshine Coast, Maroochydore, 4558, Australia \\ *Corresponding author: karen.birkenhead@research.usc.edu.au
}

\begin{abstract}
This study describes the physical activity across and within the lifetime of current ultra-endurance exercisers. Participants ( $n=115, \%$ female $=42$, age range 21 to 74 years) completed a modified version of the Lifetime Physical Activity Questionnaire (LPAQ) to assess physical activity over six life periods (5-12, 13-18, 19-30, 31-45, 46-60 and 61-75 years). Participants then were categorised into five groups according to which of these life periods they demonstrated the largest absolute increase in ultra-endurance exercise (UEE included all running and cycling) compared to the previous life period. All groups demonstrated significant increases $(p<.005)$ in UEE between their categorised life period of largest increase in UEE (IUEE) and the previous life period. Within groups, increases in average UEE MET-hours year ${ }^{-1}$ ranged from $144 \%$ to $402 \%$. Lifetime exercise volumes varied considerably within groups (average lifetime MET-hrs year ${ }^{-1}$ IUEE13-18 = $3830 \pm 2362$; IUEE19-30 = $4358 \pm 2832$; IUEE31-45 = $2975 \pm 1970$; IUEE46-60 = $2839 \pm 1721$; IUEE61-75 = $3454 \pm 1720$ ) and within the entire sample (average lifetime MET-hrs year ${ }^{-1}=3400 \pm 2229$ [range 375-12140]). This study is the first to describe the physical activity across and within the lifetime of ultra-endurance exercisers and reports that ultra-endurance exercisers exhibit significant increases in UEE within their lifetime.
\end{abstract}

Keywords: historical physical activity, ultra-marathon, ultra-cycling, ultra-triathlon, extreme exercise

Cite This Article: Karen Birkenhead, Geoff Lovell, Susan I. Barr, and Colin Solomon, "Changes in Physical Activity across the Lifetime of Current Ultra-Endurance Exercisers." Journal of Physical Activity Research, vol. 3, no. 1 (2018): 11-19. doi: 10.12691/jpar-3-1-3.

\section{Introduction}

Ultra-endurance exercise is growing in popularity worldwide with increasing numbers of people participating in long distance running and cycling events. [1,2] Ultra-endurance exercise has been defined as sustained exercise lasting longer than 6 hours and/or running distances greater than the standard marathon of $42.195 \mathrm{~km}$. [3,4] Ultra-endurance running events typically range from 50 to over $300 \mathrm{~km}$ (31 to $186 \mathrm{mi}$ ), [5] while ultra-cycling can range from 161 to $5000 \mathrm{~km}$ (100 to $3107 \mathrm{mi}$ ) in events such as the Race Across America (RAAM). [3,6,7] Ultraendurance events may take place over several days under a range of environmental conditions (e.g. altitude, temperature extremes) and on various terrains (e.g. road, mountainous, desert). [8,9,10,11] Participants engage in extended periods of exercise that is continuous or in stages and can involve one or more activities (i.e. running, cycling, swimming) [2,12].

The demographic characteristics as well as participation and performance patterns of ultra-endurance exercisers have been studied. [1,2,9,10,13,14,15,16] Research has also investigated the nutritional behaviours, [12,17,18,19,20,21] physiological responses, changes in body composition during prolonged events [5,22,23,24,25] and long term health and disease outcomes of ultra-endurance exercisers. [4] However, there is limited information on the exercise behaviours of ultra-endurance exercisers. Hoffman and Krishnam [26] reported many ultra-marathon runners engage in a lifetime of physical activity, including participation in short and ultra-distance events, with yearly running volumes ranging from 418 to more than 12,000 $\mathrm{km}$. While physical activity has been assessed across the lifespan in numerous populations, $[27,28,29,30]$ there is no detailed information specific to ultra-endurance exercisers that describes the type and volume of physical activity across the life span. The specific aim of this study was to describe the lifetime physical activity of current ultra-endurance exercisers with an emphasis on two modes of physical activity (running and cycling) specific to ultra-endurance exercisers.

\section{Materials and Methods}

\subsection{Ethics}

This study was approved by the University of the Sunshine Coast Human Research Ethics Committee. Participants were provided with an information sheet, and 
provided informed consent by completing and submitting the online questionnaires.

\subsection{Study Design}

Lifetime physical activity was investigated retrospectively using an online survey, hosted on the Survey Monkey® platform. Current male and female ultra-endurance exercisers were recruited for this study from November 2015 to June 2016. Inclusion criteria consisted of being healthy individuals, 18 years of age and older, able to complete the questionnaire in English, and being a current ultra-endurance exerciser. To qualify as a current ultra-endurance exerciser, participants had to have completed at least one ultra-endurance event within the last five years and have engaged in, on average, at least five hours of running or cycling per week during the past year.

\subsection{Participants}

Recruitment was carried out through Facebook, the Ultra Listserve (www.ultra@listserve.dartmouth.edu) and web pages associated with ultra-endurance organizations and events worldwide, including Australia, New Zealand, the United States of America and Canada. Seven hundred and eighty-one individuals started the questionnaires of which 62 were disqualified as they did not meet inclusion criteria. Two hundred and forty-eight stopped at the end of the first life period (5-12 years). One hundred and twenty completed all online questionnaires for this study.

\subsection{Questionnaires}

Information on lifetime physical activity was collected using a modified version of the Lifetime Physical Activity Questionnaire (LPAQ), [31] which is based on the original, reliable and validated version of the Historical Leisure Activity Questionnaire (HLAQ). [32] The LPAQ assesses physical activity over four life periods ranging from adolescence to older age (14-21; 22-34; 35-50 and 51-65 years). There are 26 physical activities to choose from and participants were asked to identify all those they engaged in on more than 10 occasions within each life period. Participants then estimated the total number of years, months and hours they engaged in the activity within each life period. For this study, information on physical activity was collected as part of a larger study that included lifetime dietary intake and medical health history. Rather than using two separate surveys, the diet and exercise questions were combined to facilitate data collection at the same time for each life period. The life periods in the original LPAQ were modified to match the diet history questionnaire (5-18 years; $19-30$ years; $31-45$ years; $46-60$ years and 61-75 years). [33] Also, the earliest life period was divided into 5-12 years and 13-18 years in order to capture the potential changing physical activity levels of the participants within this study who may have been involved in various forms of recreational or competitive physical activity and/or sport before the age of 18 years. To remind participants of past behaviours, cognitive prompts using autobiographical questions (e.g. where were you living? who did the cooking?) designed to place the individual in the specific time period were used. [33] This process has been shown to improve respondents' recall of details when reporting historical physical activity. [34] An additional question related specifically to cycling and running speed was also included. Participants were asked to describe their cycling and or running (if they participated in the activity) by selecting from nine options (e.g. cycling < $10 \mathrm{mph}$, to work or for pleasure; running, $5 \mathrm{mph}$ (12 $\mathrm{min} / \mathrm{mi}) ;$ I cannot remember). The LPAQ also includes questions on household activity, but for the current project, these were removed as the primary aim of this study was to assess levels of recreational activities across the lifetime. Likewise, although questions related to occupation or employment status (i.e. type and number of years in a job) were included at the end of each life period, these were not part of the calculations for lifetime physical activity. Finally, demographic information including age, sex, ethnicity and education were also collected.

Participants received a link to the first questionnaire that included the first life period (5-12 years) by e-mail, and their questionnaires for subsequent life stages were e-mailed approximately 24 hours after the previous response was received. The first questionnaire required approximately 30 minutes to complete with all subsequent questionnaires for each life period requiring approximately 10 minutes. The design and delivery of the online questionnaire over several days was intended to allow for more thought and reflection, and thereby maximise the quantity and quality of the responses.

\subsection{Missing Data}

For missing data, a standardised system was used for all participants. Across life periods, data were deleted based on the following rules: 1) If participants selected activities, but did not provide information on years, months or hours, 2) If the number of years participating in an activity were missing, 3) If information was missing for months and/or hours of cycling or running and the participant reported "no" for the additional question related to these activities, and 4) If participants intermittently or consistently missed information across consecutive life periods. Following these rules, less than $1.5 \%$ of data were deleted across life periods from 5-12 through to 46-60 years. For all remaining missing data, the mean was inserted for those participants who provided information on the number of years, but were missing months and/or hours of an activity (38 data points or $<0.5 \%$ of total data). If data on speed was missing for running and cycling, the level reported by the largest number of participants during each life period was used.

\subsection{Assessment of Lifetime Physical Activity}

Physical activity was calculated as the total number of hours spent in each activity per life period. This was determined by multiplying the number of years, by the number of months and the number of hours per week spent in each activity. Months were converted to weeks by multiplying by 4.35 . Each activity was multiplied by the intensity according to the metabolic equivalent (MET) as determined by the Compendium of Physical Activities. [35] All activities were summed to obtain a total number 
of MET-hours of activity for each life period and the yearly average was calculated by dividing by the number of years spent in the life period. Finally, lifetime total average MET-hours per week and year were calculated by dividing the total lifetime MET-hours by the total number of weeks or years spent in all life periods based on the participant's current age.

\subsection{Categorising the Lifetime Physical Activity of Ultra-endurance Exercisers}

It was evident from the raw data that the participants engaged in various aerobic (including a variety of endurance sports such as running, cycling, swimming and cross-country skiing) and non-aerobic/strength-based physical activities across the participant's current lifespan. Therefore, the approach used to categorise the ultra-endurance exercisers was conducted in three stages in order to ensure the physical activity of the participants was described appropriately. The first stage involved graphing average MET-hours per year for all physical activities for each participant across all life periods. All activities were also graphed according to type (i.e. total, running and cycling, all other aerobic activities and all other non-aerobic or strength activities). From these graphs, it was evident the ultra-endurance exercisers had a diverse physical activity background across the lifetime as exercise volume increased, decreased or remained approximately the same from one life period to the next. As such, a decision was made to describe participants based on ultra-endurance exercise (UEE), defined as all running and cycling, as opposed to all other physical activities. This was based on the premise that the period during which participants had the largest increase in running and cycling coincided with a decision to become an ultra-endurance exerciser and, as such, these two forms of physical activity would be the predominant exercise choice. Furthermore, the decision to categorise participants based on running and cycling considered criteria for taking part in this study, which included being a current ultra-endurance runner and/or cyclist. The second stage involved calculating the difference across each life period in average running and cycling MET-hours per year and grouping participants based on similar changes from one life period to the next. The third stage was to use the largest overall increase in average running and cycling MET-hours per year between consecutive life periods as the rule for grouping participants into one of five, increase in ultra-endurance exercise (IUEE), groups. The decision to use running and cycling was supported when visual inspection of the bar graphs that included activities grouped according to type (i.e. total, running and cycling, all other aerobic activities and all other non-aerobic or strength activities) showed all other forms of aerobic exercise primarily decreased during the period in which UEE had the largest increase. This approach to processing the data was necessary due to the considerable age range (21 to 74 years) and the varying times at which participants may have started ultra-endurance exercise. The method allowed this group of ultra-endurance exercisers to be described based entirely on the largest increase in running and cycling within their lifetime.

\subsection{Statistical Analysis}

Descriptive statistics are displayed as mean and standard deviation. Paired sample t-tests were used to compare differences in average MET-hours per year of physical activity between the life period within each IUEE group and the preceding life period. Statistical analyses were performed using SPSS version 24.0 for windows with the alpha level set at $p<.05$.

\section{Results}

\subsection{Participant Characteristics}

Within each life period there were 81 data points per participant and a maximum total number of points (including all participants) of 44,469. There were 45 participants with missing exercise data (37.5\%); missing data ranged from one to 106 data points and from 0 to $2 \%$ across all life periods. Participant characteristics are presented in Table 1. Five participants were removed from the IUEE groups due to missing data. Ninety-seven percent (112 of 115) of participants were ultra-runners and/or ultra-cyclists (two were ultra-walkers and one was an ultra-triathlete). Forty-one percent of ultra-runners did not report any cycling within the IUEE group they were categorised, while $47 \%$ of ultra-cyclists did not report any running within the IUEE group they were categorised. The majority of participants were Caucasian (91\%) and had a bachelor's degree or higher (71\%). Forty-nine percent were from Australia, 47\% were from North America and $4 \%$ were from other countries. Fifty-eight percent of participants were men. Eighty-three percent of participants indicated they started training for ultra-endurance exercise from one to 12 months before they completed their first event. Seven percent started training from 13 months to four years before their first event. Ten percent started training after they completed their first ultra-endurance event with most indicating they had been previously training for various short and long-distance running, cycling or triathlon events.

\subsection{Ultra-endurance Exercise Groups}

Participant groups based on the largest increase in average MET-hours per year for running and cycling are presented in Figure 1. The proportion of total average MET-hours per year contributed by running and cycling, other aerobic activities and all other activities are presented in Figure 2. The highest percentage of total MET-hours per year for each group was made up of ultra-endurance type activities (running and cycling). There was a significant increase (Figure 2) in average MET-hours per year for running and cycling between the life period in which participants were categorised and the preceding life period (i.e. between 5-12 years and 13-18 years for IUEE13-18). Average running and cycling MET-hrs per year increased from 1230 to 3389 (176\%) for IUEE13-18; from 1052 to 4560 (333\%) for IUEE19-30; from 931 - 4676 (402\%) for IUEE31-45; from 1305 to 5756 (341\%) for IUEE46-60 and from 4218 to 10293 (144\%) for IUEE61-75. There was a significant decrease $(p=.028)$ in average MET 
hours per year between these two life periods for all other aerobic activities for IUEE19-30. For all other IUEE groups, there was no significant difference in average
MET hours per year between these two life periods for all other aerobic activities (IUEE13-18 $p=.171$; IUEE31-45 $p=.075$; IUEE46-60 $p=.626$; IUEE61-75; $p=.505)$.

(a)

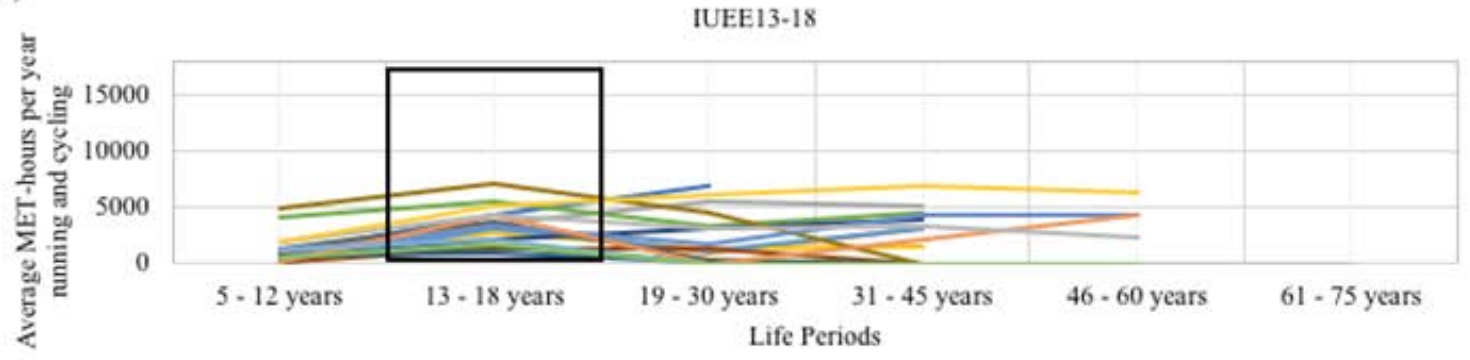

(b)

IUEE19-30

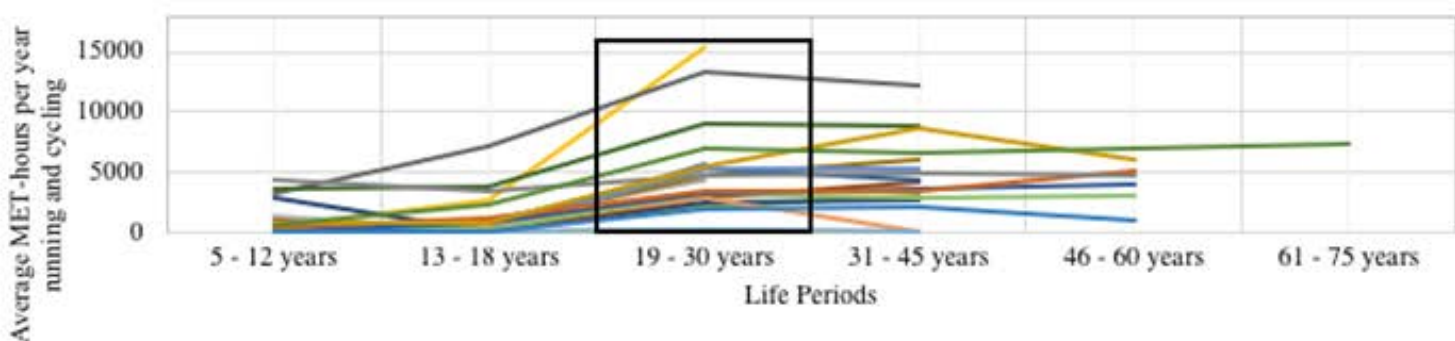

(c)

IUEE $31-45$

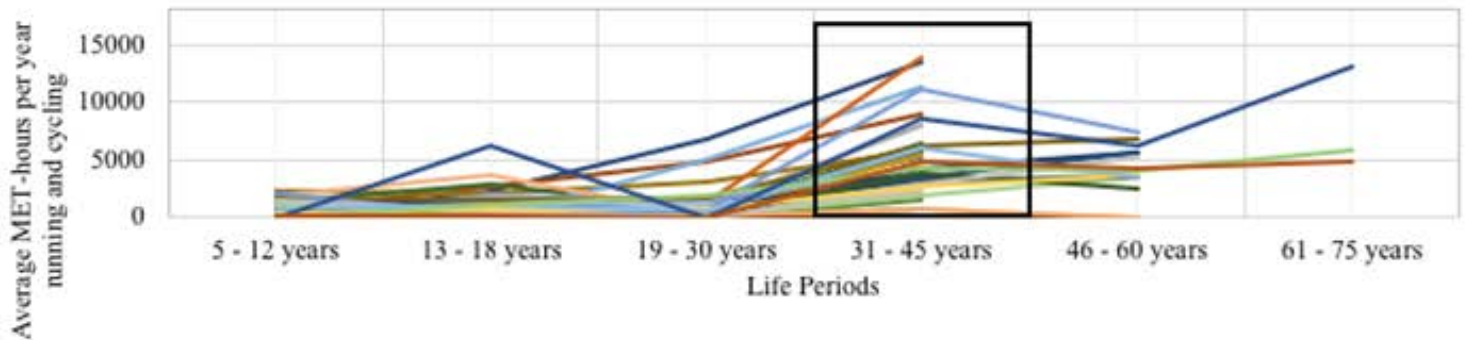

(d)

IUEE46-60

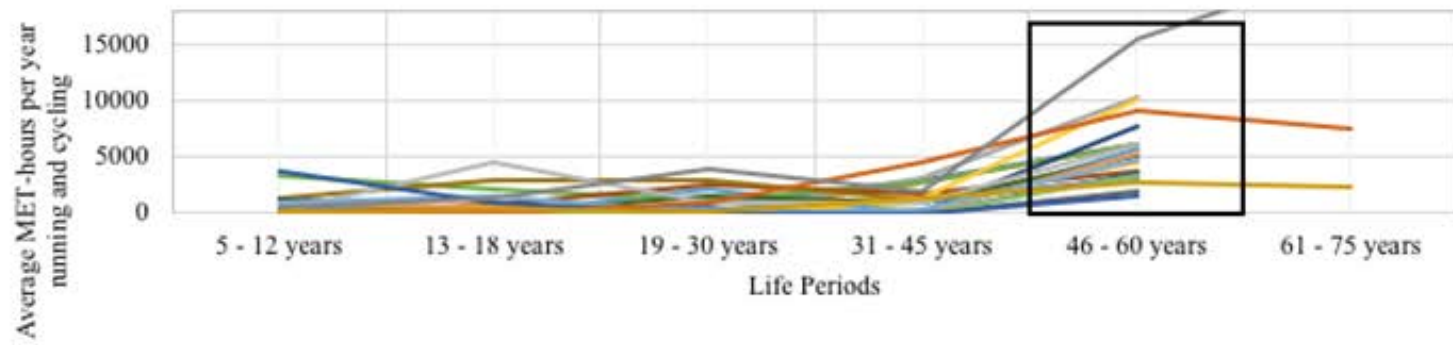

(e)

IUEE61-75

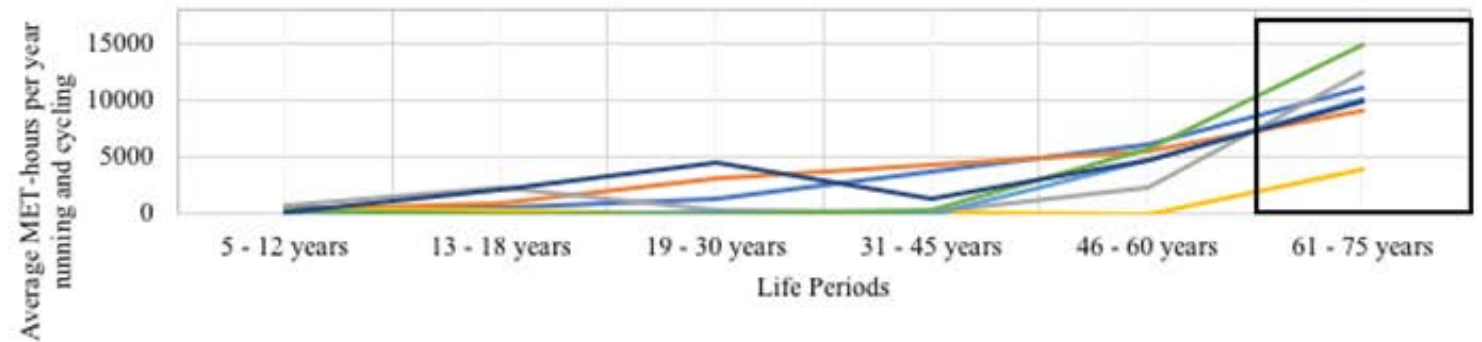

Figure 1. Increase in ultra-endurance exercise (IUEE) groups based on the largest increase between life periods in average running and cycling METhours per year. Participants in IUEE13-18 (a) showed the largest increase in average MET-hours per year in running and cycling in the life period for 13-18 years; IUEE19-30 (b) in the life period for 19-30 years; IUEE31-45 (c) in the life period for 31-45 years; IUEE46-60 (d) in the life period for 4660 years and IUEE61-75 (e) in the life period for 61-75 years. Each line represents one participant with boxed area highlighting the life period with the largest increase in MET-hours 
(a)

IUEE13-18
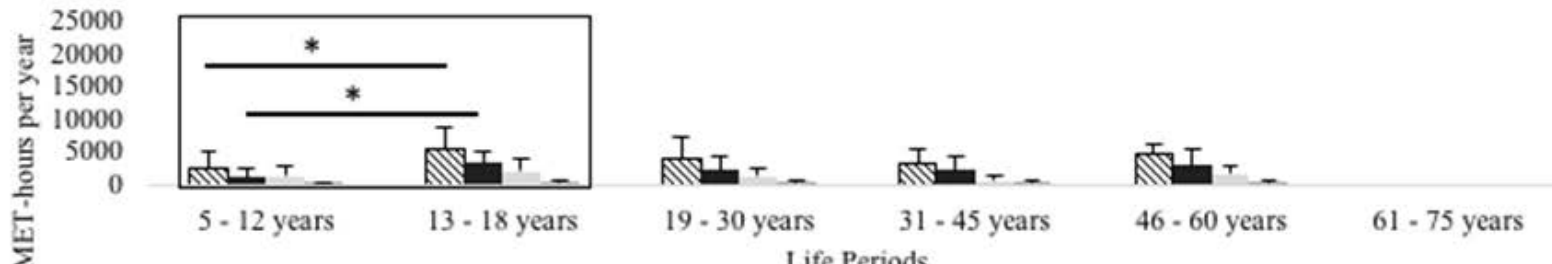

Life Periods

QTotal $\quad$ Running and Cycling $=$ All other aerobic activities $\equiv$ All other non aerobic or strength activities

(b)

IUEE19-30

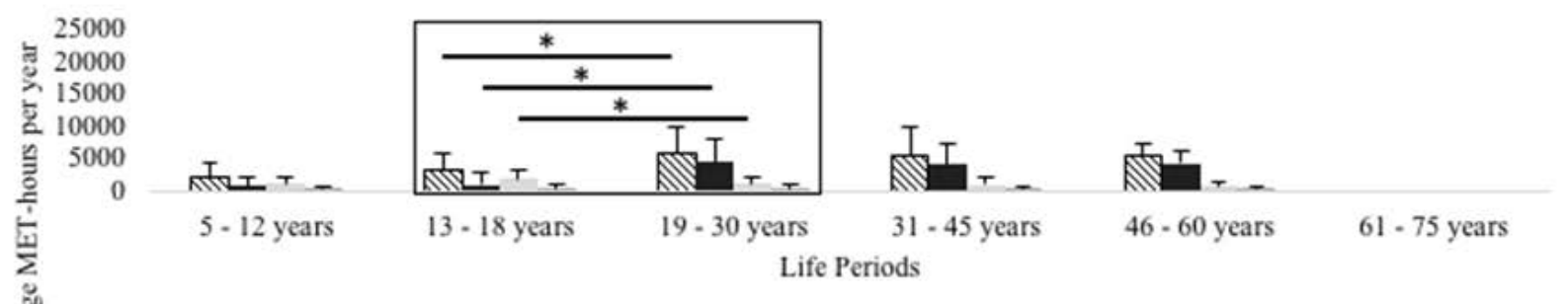

QTotal $\quad$ Running and Cycling $=$ All other aerobic activities $=$ All other non aerobic or strength activities

(c)

IUEE31-45

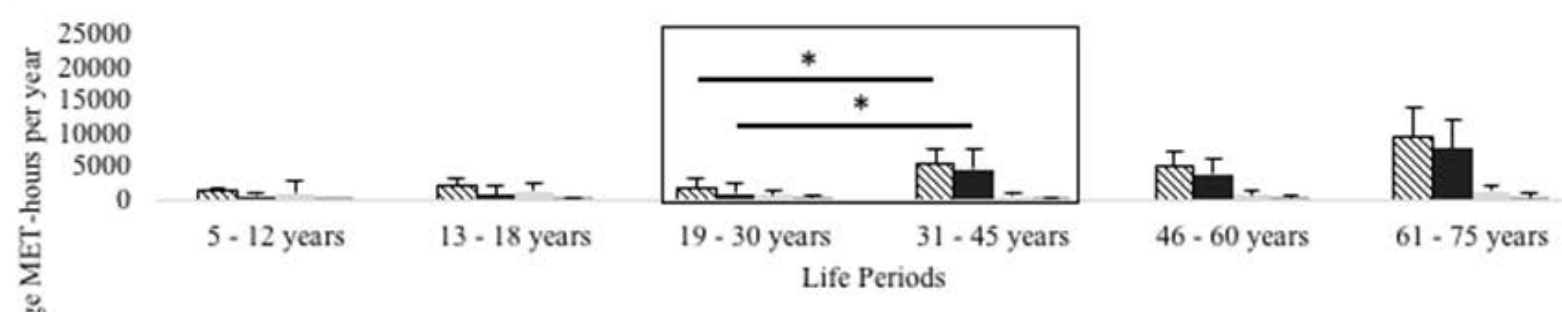

QTotal $\quad$ Running and Cycling $=$ All other aerobic activities $\quad$ All other non aerobic or strength activities

(d) IUEE46-60

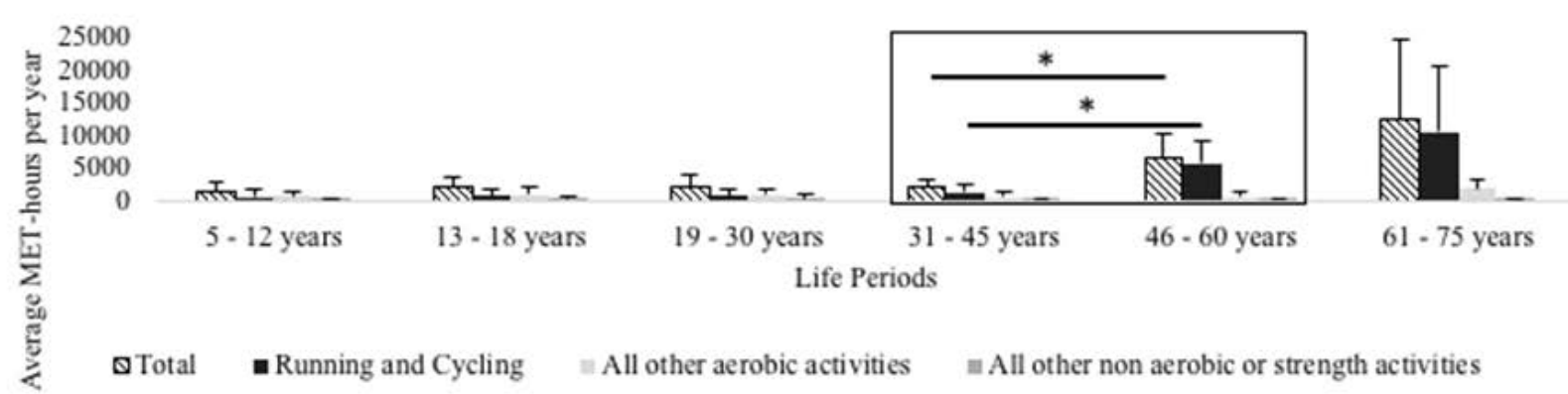

(e)

\section{IUEE61-75}
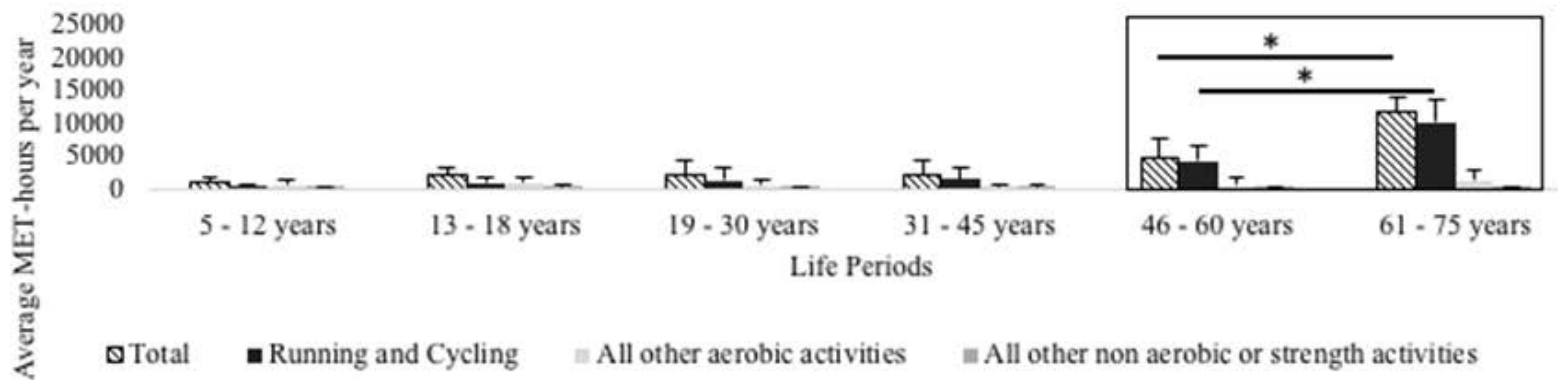

QTotal = Running and Cycling $=$ All other aerobic activities $=$ All other non aerobic or strength activities

Figure 2. Proportion of total average MET-hours per year contributed by running and cycling and other activities across the life periods for IUEE13-18 (a), IUEE19-30 (b), IUEE31-45 (c), IUEE46-60 (d) and IUEE61-75 (e). Error bars represent SD and * indicates a significant difference between life periods $(p<.05)$. Note: Visually the largest increase for IUEE31-45 (c) and IUEE46-60 (d) appear in later life periods. This is due to reduced participant numbers in subsequent life periods increasing the average for the group (i.e. for IUEE31-45 $n=44$ in 31-45 years and $n=3$ in $61-75$ years; for IUEE46-60 $n=22$ in 46-60 years and $n=3$ in 61- 75 years) 
Table 1. Participant characteristics according to increase in ultra-endurance exercise (IUEE) group ${ }^{\mathrm{a}}$

\begin{tabular}{|c|c|c|c|c|c|c|}
\hline Participants & $\begin{array}{c}\text { All } \\
\mathrm{n}=115\end{array}$ & $\begin{array}{c}\text { IUEE13-18 }^{b} \\
n=18\end{array}$ & $\begin{array}{c}\text { IUEE19-30 } \\
n=24\end{array}$ & $\begin{array}{c}\text { IUEE31-45 } \\
\mathrm{n}=44\end{array}$ & $\begin{array}{c}\text { IUEE46-60 } \\
\mathrm{n}=22\end{array}$ & $\begin{array}{c}\text { IUEE61-75 } \\
n=7\end{array}$ \\
\hline Age range (yr) & $21-74$ & $25-60$ & $21-64$ & $32-70$ & $46-70$ & $61-74$ \\
\hline \multicolumn{7}{|l|}{ Mean age (yr) } \\
\hline Both & $46.6 \pm 10.9$ & $43.6 \pm 8.0$ & $40.1 \pm 11.0$ & $44.2 \pm 9.0$ & $54.9 \pm 5.8$ & $64.9 \pm 4.7$ \\
\hline Men & $49.3 \pm 11.8$ & $44.4 \pm 9.5$ & $40.1 \pm 13.7$ & $47.0 \pm 9.7$ & $57.1 \pm 5.9$ & $65.5 \pm 4.8$ \\
\hline Women & $42.7 \pm 8.0$ & $42.3 \pm 5.4$ & $40.1 \pm 9.1$ & $40.2 \pm 6.2$ & $50.9 \pm 2.7$ & $\mathrm{n} / \mathrm{a}$ \\
\hline \multicolumn{7}{|c|}{ Average age at first ultrac ${ }^{c}(y r)$} \\
\hline Both & $\begin{array}{c}38.2 \pm 10.2 \\
(\mathrm{n}=77)\end{array}$ & $\begin{array}{c}34.3 \pm 9.2 \\
(n=13)\end{array}$ & $\begin{array}{c}33.3 \pm 8.3 \\
(\mathrm{n}=15)\end{array}$ & $\begin{array}{c}36.8 \pm 8.0 \\
(n=30)\end{array}$ & $\begin{array}{c}46.4 \pm 9.9 \\
(n=14)\end{array}$ & $\begin{array}{c}44.8 \pm 16.5 \\
(\mathrm{n}=5)\end{array}$ \\
\hline Men & $\begin{array}{l}39.5 \pm 11.7 \\
(n=47)\end{array}$ & $\begin{array}{l}34.9 \pm 10.0 \\
(\mathrm{n}=8)\end{array}$ & $\begin{array}{c}30.7 \pm 10.3 \\
(\mathrm{n}=7)\end{array}$ & $\begin{array}{c}39.5 \pm 8.1 \\
(\mathrm{n}=19)\end{array}$ & $\begin{array}{c}47.9 \pm 12.2 \\
(\mathrm{n}=9)\end{array}$ & $\begin{array}{l}45.5 \pm 19.0 \\
(\mathrm{n}=4)\end{array}$ \\
\hline Women & $\begin{array}{c}36.2 \pm 7.0 \\
(\mathrm{n}=30)\end{array}$ & $\begin{array}{c}33.4 \pm 8.8 \\
(\mathrm{n}=5)\end{array}$ & $\begin{array}{c}35.6 \pm 5.9 \\
(\mathrm{n}=8)\end{array}$ & $\begin{array}{c}33.8 \pm 6.5 \\
(n=11) \\
\end{array}$ & $\begin{array}{c}43.8 \pm 2.2 \\
(n=5)\end{array}$ & n/a \\
\hline Sex & $\begin{array}{l}M=67 \\
F=48\end{array}$ & $\begin{aligned} \mathrm{M} & =11 \\
\mathrm{~F} & =7\end{aligned}$ & $\begin{array}{l}M=10 \\
F=14\end{array}$ & $\begin{array}{l}M=26 \\
F=18\end{array}$ & $\begin{aligned} \mathrm{M} & =14 \\
\mathrm{~F} & =8\end{aligned}$ & $\begin{array}{l}M=6 \\
F=1\end{array}$ \\
\hline \multicolumn{7}{|c|}{ Ultra-endurance type (\%) } \\
\hline Ultra running $^{\mathrm{d}}$ & $81(70)$ & $15(83)$ & $15(62)$ & $32(73)$ & $14(64)$ & $5(71.4)$ \\
\hline Ultra-cycling & 17 (15) & $1(6)$ & $4(17)$ & $5(11)$ & $6(27)$ & $1(14.3)$ \\
\hline Combination $^{e}$ & $17(15)$ & $2(11)$ & $5(21)$ & $7(16)$ & $2(9)$ & $1(14.3)$ \\
\hline \multicolumn{7}{|c|}{$\begin{array}{l}\text { Average Lifetime } \\
\text { MET-hours per year }\end{array}$} \\
\hline Men & $3538 \pm 2221$ & $4367 \pm 2675$ & $4444 \pm 2379$ & $3162 \pm 2068$ & $3039 \pm 2079$ & $3301 \pm 1832$ \\
\hline Women & $3209 \pm 2248$ & $2985 \pm 1585$ & $4296 \pm 3203$ & $2706 \pm 1842$ & $2489 \pm 799$ & $\mathrm{n} / \mathrm{a}$ \\
\hline \multicolumn{7}{|c|}{ Average Lifetime } \\
\hline Men & $68.0 \pm 42.7$ & $84.0 \pm 51.4$ & $85.5 \pm 45.7$ & $60.8 \pm 39.8$ & $58.4 \pm 40.0$ & $63.5 \pm 35.2$ \\
\hline Women & $61.7 \pm 43.2$ & $57.4 \pm 30.5$ & $82.6 \pm 61.6$ & $52.0 \pm 35.4$ & $47.9 \pm 15.4$ & $\mathrm{n} / \mathrm{a}$ \\
\hline \multicolumn{7}{|c|}{ Life period when completed first ultra (\%) } \\
\hline $19-30$ years & $18(16)$ & $3(17)$ & $6(25)$ & $7(16)$ & $1(4.5)$ & $1(14)$ \\
\hline $31-45$ years & $47(41)$ & $8(44)$ & $9(38)$ & $21(48)$ & $7(32)$ & $2(29)$ \\
\hline $46-60$ years & $13(11)$ & $1(6)$ & $1(4)$ & $4(9)$ & $6(27)$ & $1(14)$ \\
\hline $61-75$ years & $2(2)$ & 0 & 0 & 0 & $1(4.5)$ & $1(14)$ \\
\hline Unknown & $35(30)$ & $6(33)$ & $8(33)$ & $12(27)$ & $7(32)$ & $2(29)$ \\
\hline
\end{tabular}

${ }^{\mathrm{a}}$ Data are presented as mean \pm SD for continuous variables and $\mathrm{n}(\%)$ for categorical variables.

${ }^{\mathrm{b}}$ IUEE13-18 through IUEE61-75 correspond to ultra-endurance exercise groups 13-18, 19-30, 31-45, 46-60, 61-75 years

${ }^{\mathrm{c}} 77$ participants reported age at first ultra

dincludes ultra-walking

e includes ultra-running, ultra-cycling, and ultra-triathlon or combination of all three

\subsection{Physical Activity in the Life Periods Following the Largest Increase in Average MET-hours}

Fifty-two percent of participants (60 of 115) showed their largest increase in UEE in the life period that includes their current age and, among this group, 57\% (34 of 60) also completed their first ultra in the IUEE group that includes their current age. Overall, 30\% of participants completed their first ultra in their categorised IUEE period. Eighteen percent of participants completed their first ultra in a life period before their categorised IUEE period, while another 23\% completed their first ultra in a life period following their categorised IUEE period. Nine percent did not indicate when they completed their first ultra, but reported an increase in running, cycling and/or triathlon activity. Twenty percent did not provide any information on when they completed their first ultra or any changes in UEE activities. Among participants whose current age was older than their categorised IUEE group ( $\mathrm{n}=55), 11 \%(\mathrm{n}=6), 31 \%$ $(n=17)$ and $58 \%(n=32)$ had no change, increased or decreased ultra-endurance activity after their largest increase, respectively. Of the 32 participants who showed a decrease after their categorised IUEE period, 53\% continued to decrease, $31 \%$ decreased between life periods and then increased to the life period that includes their current age, $16 \%$ increased and then decreased to the life period that includes their current age.

\section{Discussion}

This study was designed, and is the first, to investigate and quantify the lifetime physical activity of current ultra-endurance exercisers across multiple life periods. The aim was to describe both the type and volume of physical activities, with the primary interest being in ultraendurance exercise. This was achieved by gathering detailed information on the frequency, duration and intensity of physical activities from childhood to current age. A novel approach was used to divide this heterogeneous population into distinct groups based on the largest increase in ultra-endurance exercise (running and cycling) across the lifetime.

The IUEE groups in this study were similar to other published reports with ultra-endurance exercisers. [4,26,36] Consistent with previous research, the participants (both within IUEE groups and as a whole) were highly educated and mostly Caucasian. [26,36] With the exception of the 
IUEE19-30 group, there were more ultra- endurance men than women (Table 1), which has been consistently reported in previous studies. [1,2,3,13,15,20,36,37] Likewise, similar to previous research, $[14,36,38]$ the average age of men was higher than women for all participants (Table 1).

This study used a self-administered, online questionnaire to investigate the lifetime physical activity of a unique population who engage in prolonged periods of physical activity. Previous research has investigated lifetime physical activity using various measurement tools [28,39] and reported findings in several ways (i.e. MET-hrs week ${ }^{1}$ grouped into quintiles from low to high; time in hours per week; intensity level) $[30,40,41]$ making it difficult to make direct comparisons with the results from this study. In general, the volume of total recreational physical activity reported by ultra-endurance exercisers was higher than general populations. [29,42,43] This was found for both men and women across all IUEE groups in the present study. The ultra-endurance women (age range of 25-61 years) had higher average lifetime MET-hours per week compared to postmenopausal women (aged $\geq 50$ years) and female college alumnae (age range of 39-50 years) (lifetime average ranged from 48-83 MET-hours week $^{-1}$ across all IUEE groups vs $<20$ and 35 lifetime MET-hours week ${ }^{-1}$ for postmenopausal women and female college alumnae, respectively). [29,31] Likewise, the ultra-endurance men in this study (age range of 21-74 years) had higher average lifetime MET-hours per week compared to healthy men (aged $\geq 50$ years) (average ranged from 58-86 MET-hours week ${ }^{-1}$ across all IUEE groups vs $\sim 26$ to $<40$ MET-hours week $^{-1}$ for recreational/leisure activities of healthy men). [28,42] Overall, these findings suggest ultra-endurance exercisers participate in higher volumes of recreational or leisure type physical activities across the lifetime than general populations.

This study grouped participants according to the largest overall increase in average MET-hours per year for running and cycling between consecutive life periods. All of the participants in this study, the majority (97\%) of whom self-identified as an ultra-runner and/or ultra-cyclist, completed an ultra-endurance event within the last five years. Categorising participants according to a significant change in running and cycling, as opposed to total physical activity, provided a new method of characterising a group of individuals who all identified as current ultra-endurance exercisers. There is limited research investigating the reasons people take part in ultra-endurance exercise or at what stage an individual identifies as an ultra-endurance exerciser. Ultra-marathoners reported health, personal goals and self-esteem as primary reasons for participating in ultra-endurance running. [44,45] Although only $30 \%$ of participants in this study (of the $70 \%$ who reported this information) completed their first ultra in their categorised IUEE group, it is possible the increase in exercise volume was associated with the time period they became an ultra-endurance exerciser. This is supported by information provided by participants on when they started training for UEE with most (87\% of those who responded) starting approximately two years before completing their first ultra-endurance event. As shown in Figure 1, changes in physical activity following the largest increase are varied, with some participants increasing, decreasing or remaining about the same. Ultra-endurance exercise requires a consistent and committed effort to maintain cardiovascular fitness. Openended responses (data not shown) from the ultraendurance exercisers in this study suggest some of the reasons for a decrease in physical activity following the largest increase could be due to personal (depression or job change) or family issues (divorce, separation). Other reasons could be having children or personal injury, which are also reasons for a decrease in running previously reported by ultramarathon runners. [26] Overall, this study demonstrates the volume of physical activity of current ultra-endurance exercisers is highly varied across the lifetime and that this group demonstrated significant increases in running and/or cycling at some stage within their lifetime.

\subsection{Limitations}

This study has six known potential limitations. Firstly, the approach for grouping individuals is limited by the age of the participant. Although the IUEE13-18 group includes participants ranging in age from 25-60 years, it was not possible to group younger participants in older life periods simply due to their age. Secondly, this study may be limited by self-selection bias and small sample size, particularly in the oldest age category. Thirdly, the data for this study were self-reported from the past and are limited by participants' recall from up to several decades ago. However, steps were taken to enhance recall with the use of cognitive prompts. Fourthly, respondents were asked to report information that approximates physical activity over life periods that included a span of several years and information on year to year variation is missing. Fifthly, the questionnaire used in this study was found to be highly reproducible in a population of college age women and therefore, may not be directly applicable to the group of highly active individuals in this study. Also, this study did not validate the online, modified version of the lifetime physical activity questionnaire. However, previous research has shown the recall of historical physical from several years ago can produce reasonably accurate responses. [34,46] Finally, it is possible the average MET-hours per year were underestimated due to the rules for missing data whereby some data values were assigned a zero value.

\section{Conclusions}

This study used a new and unique approach to describe the lifetime physical activity of a group of current ultra-endurance exercisers. The results illustrate that this group, although similar based on participation in ultraendurance exercise, has a varied and diverse physical activity background across the lifetime. The approach used to categorise the ultra-endurance exercisers was based on a specific life period with the largest increase in exercise volume and it is difficult to characterise the participants following this initial increase, as there were no consistent changes (and many individuals were still in the life period with the largest increase in exercise volume). 
The ultra-endurance exercisers in this study engaged in, on average, higher volumes of physical activity throughout life compared to the general population. There is a need to develop a physical activity measure specific to ultra-endurance exercisers to better describe the exercise behaviours of this population across the lifetime. Specifically, questions related to lifetime training and competing, motivation for participating in ultra-endurance exercise and reasons for changes in activity across the lifetime would be useful. As participation in ultra-endurance exercise continues to increase, more research is needed to understand those who engage in this specific form of physical activity.

\section{List of Abbreviations}

HLAQ: Historical Leisure Activity Questionnaire

IUEE: Increase in Ultra-Endurance Exercise

LPAQ: Lifetime Physical Activity Questionnaire

MET: Metabolic Equivalents

MET-hr-week : Metabolic Equivalent hours per week

MET-hr ${ }^{-y r}$ : Metabolic Equivalent hours per year

UEE: Ultra-Endurance Exercise

UEE MET-hr ${ }^{-y r}$ : Ultra-Endurance Exercise Metabolic Equivalent hours per year

\section{Acknowledgements}

No funding was used for this project and there are no conflicts of interest.

\section{References}

[1] Cejka, N., Rust, C. A., Lepers, R., Onywera, V., Rosemann, T. and Knechtle, B., "Participation and performance trends in 100km ultra-marathons worldwide," Journal of Sports Sciences, 32 (4), 354-366, 2014.

[2] Rüst, C. A., Knechtle, B., Knechtle, P., Rosemann, T., and Lepers, R., "Participation and performance trends in triple iron ultratriathlon - a cross-sectional and longitudinal data analysis," Asian Journal of Sports Medicine, 3 (3), 145-152, Sep, 2012.

[3] Shoak, M. A., Knechtle, B., Knechtle, P., Rust, C. A., Rosemann, T., and Lepers, R., "Participation and performance trends in ultracycling," Open Access Journal of Sports Medicine, 4, 41-51, 2013.

[4] Hoffman, M. D. and Krishnan, E., "Health and exercise-related medical issues among 1,212 ultramarathon runners: Baseline findings from the ultrarunners longitudinal tracking (ULTRA) study," Plos One, 9 (1), e83867, Jan 8, 2014.

[5] Vernillo, G. et al., "Changes in lung function during an extreme mountain ultramarathon," Scandinavian Journal of Medicine and Science in Sports, 25 (4), e374-380, Aug, 2015.

[6] Knechtle, B., Wirth, A., Knechtle, P., Ruest, C. A., and Rosemann, T., "A comparison of ultra-endurance cyclists in a qualifying ultracycling race for Paris-Brest-Paris and race across America-Swiss cycling marathon," Perceptual and Motor Skills, 114 (1), 96-110, Feb, 2012.

[7] Armstrong, L. E. et al., "Nutritional, physiological, and perceptual responses during a summer ultraendurance cycling event," Journal of Strength and Conditioning Research, 26 (2), 307-318, 2012.

[8] Saugy, J., Place, N., Millet, G. Y., Degache, F., Schena, F., and Millet, G. P., "Alterations of neuromuscular function after the world's most challenging mountain ultra-marathon," PLoS One, 8 (6), e65596, 2013.

[9] Gloor, R. U. et al., "Sex-related trends in participation and performance in the 'Swiss bike masters' from 1994 - 2012," Perceptual and Motor Skills, 116 (2), 640-654, Apr, 2013.
[10] da Fonseca-Engelhardt, K., Knechtle, B., Rust, C. A., Knechtle, P., Lepers, R., and Rosemann, T., "Participation and performance trends in ultra-endurance running races under extreme conditions 'Spartathlon' versus 'Badwater'," Extreme Physiology and Medicine, 2 (1), 15, 2013.

[11] Knoth, C., Knechtle, B., Rust, C. A., Rosemann, T., and Lepers, R., "Participation and performance trends in multistage ultramarathons-the 'Marathon des Sables' 2003-2012," Extreme Physiology and Medicine, 1 (1), 13, 2012.

[12] Costa, R. J. S. et al., "Water and sodium intake habits and status of ultra-endurance runners during a multi-stage ultra-marathon conducted in a hot ambient environment: an observational field based study," Nutrition Journal, 12 13, 2013.

[13] Ehrensperger, L., Knechtle, B., Rüst, C. A., and Rosemann, T., "Participation and performance trends in 6-hour ultra-marathoners - A retrospective data analysis of worldwide participation from 1991-2010," Journal of Human Sport and Exercise, 8 (4), 905-924, 2013.

[14] Hoffman, M. D. and Wegelin, J. A., "The western states 100-Mile endurance run: Participation and performance trends," Medicine and Science in Sports and Exercise, 41 (12), 2191-2198, Dec, 2009.

[15] Sehovic, E., Knechtle, B., Rüst, C. A., and Rosemann, T., "12hour ultra-marathons - Increasing worldwide participation and dominance of Europeans," Journal of Human Sport and Exercise, 8 (4), 932-953, 2013.

[16] Knechtle, B., Rüst, C. A., Rosemann, T., and Lepers, R., "Agerelated changes in 100-km ultra-marathon running performance," Age, 34 (4), 1033-1045, Aug, 2012.

[17] Costa, R. J. S., Gill, S. K., Hankey, J., Wright, A., and Marczak, S., "Perturbed energy balance and hydration status in ultra-endurance runners during a $24 \mathrm{~h}$ ultra-marathon," British Journal of Nutrition, 112 (3), 428-437, 2014.

[18] Fallon, K. E., Broad, E., Thompson, M. W., and Reull, P. A., "Nutritional and fluid intake in a 100-km ultramarathon," International Journal of Sport Nutrition and Exercise Metabolism, 8 (1), 24-35, 1998.

[19] Peters, E. M. and Goetzsche, J. M., "Dietary practices of South African ultradistance runners," International Journal of Sport Nutrition and Exercise Metabolism, 7 (2), 80-103, 1997.

[20] Wardenaar, F. C. et al., "Nutrient intake by ultramarathon runners: Can they meet recommendations?," International Journal of Sport Nutrition and Exercise Metabolism, 25 (4), 375-386, Aug, 2015.

[21] Dempster, S., Britton, R., Murray, A., and Costa, R. J. S., "Case study: Nutrition and hydration status during $4,254 \mathrm{~km}$ of running over 78 consecutive days," International Journal of Sport Nutrition and Exercise Metabolism, 23 (5), 533-541, Oct, 2013.

[22] Bischof, M., Knechtle, B., Rust, C. A., Knechtle, P., and Rosemann, T., "Changes in skinfold thicknesses and body fat in ultra-endurance cyclists," Asian Journal of Sports Medicine, 4 (1), 15-22, Mar, 2013.

[23] Bircher, S., Enggist, A., Jehle, T., and Knechtle, B., "Effects of an extreme endurance race on energy balance and body composition A case study," Journal of Sports Science and Medicine, 5 (1), 154-162, 2006.

[24] Schuetz, U. H. W. et al., "Characteristics, changes and influence of body composition during a $4486 \mathrm{~km}$ transcontinental ultramarathon: Results from the transeurope footrace mobile whole body MRI-project," BMC Medicine, 11 (122), May 2013.

[25] Hill, R. J. and Davies, P. S. W., "Energy expenditure during 2 wk of an ultraendurance run around Australia," Medicine and Science in Sports and Exercise, 33 (1), 148-151, 2001.

[26] Hoffman, M. D. and Krishnan, E., "Exercise behavior of ultramarathon runners: Baseline findings from the ultra study," Journal of Strength and Conditioning Research, 27 (11), 29392945, Nov, 2013.

[27] Middlekauff, M., Lee, W., Egger, M. J., Nygaard, I. E., and Shaw, J. M., "Physical activity patterns in healthy middle-aged women," Journal of Women and Aging, 28 (6), 469-476, 2016.

[28] Boisvert-Vigneault, K., Payette, H., Audet, M., Gaudreau, P., Bélanger, M., and Dionne, I. J., "Relationships between physical activity across lifetime and health outcomes in older adults: Results from the NuAge cohort," Preventive Medicine, 91 37-42, Oct, 2016.

[29] Tam, C. et al., "Lifetime physical activity in postmenopausal Caucasian and Chinese-Canadian women," European Journal of Cancer Prevention, 23 (2), 90-95, Mar, 2014. 
[30] Sormunen, J. et al., "Lifetime physical activity and cancer incidence--a cohort study of male former elite athletes in Finland," Journal of Science and Medicine in Sport, 17 (5), 479-484, Sep, 2014.

[31] Chasan-Taber, L., Erickson, J. B., McBride, J. W., Nasca, P. C., Chasan-Taber, S., and Freedson, P. S., "Reproducibility of a self-administered lifetime physical activity questionnaire among female college alumnae," American Journal of Epidemiology, 155 (3), 282-289, 2002.

[32] Kriska, A. M., Sandler, R. B., Cauley, J. A., LaPorte, R. E., Hom, D. L., and Pambianco, G., "The assessment of historical physical activity and its relation to adult bone parameters," American Journal of Epidemiology, 127 (5), 1053-1063, 1988.

[33] Hosking, D., Danthiir, V., Nettelbeck, T., and Wilson, C., "Assessing lifetime diet: Reproducibility of a self-administered, non-quantitative FFQ," Public Health Nutrition, 14 (5), 801-808, 2011.

[34] Friedenreich, C. M., Courneya, K. S., and Bryant, H. E., "The lifetime total physical activity questionnaire: development and reliability," Medicine and Science in Sports and Exercise, 30 (2), 266-274, Feb, 1998.

[35] Ainsworth, B. E. et al., "2011 compendium of physical activities: A second update of codes and MET values," Medicine and Science in Sports and Exercise, 43 (8), 1575-1581, 2011.

[36] Hoffman, M. D. and Fogard, K., "Demographic characteristics of 161-km ultramarathon runners," Research in Sports Medicine, 20 (1), 59-69, 2012.

[37] Hoffman, M. D., Ong, J. C., and Wang, G., "Historical analysis of participation in $161 \mathrm{~km}$ ultramarathons in North America," International Journal of the History of Sport, 27 (11), 1877-1891, 2010.

[38] Tokudome, S. et al., "Anthropometric, lifestyle and biomarker assessment of Japanese non-professional ultra-marathon runners," Journal of Epidemiology, 14 (5), 161-167, Sep, 2004.
[39] Kim, S., So, W. Y., Kim, J., and Sung, D. J., "Relationship between bone-specific physical activity scores and measures for body composition and bone mineral density in healthy young college women," PLoS One, 11 (9), e0162127, 2016.

[40] Rideout, C. A., McKay, H. A., and Barr, S. I., "Self-reported lifetime physical activity and areal bone mineral density in healthy postmenopausal women: The importance of teenage activity," Calcified Tissue International, 79 (4), 214-222, 2006.

[41] Si, S., Boyle, T., Heyworth, J., Glass, D. C., Saunders, C., and Fritschi, L., "Lifetime physical activity and risk of breast cancer in pre-and post-menopausal women," Breast Cancer Research and Treatment, 152 (2), 449-462, Jul, 2015.

[42] Gill, S. J. et al., "Association between lifetime physical activity and cognitive functioning in middle-aged and older community dwelling adults: Results from the brain in motion study," Journal of the International Neuropsychological Society, 21 (10), 816-830, Nov, 2015.

[43] De Vera, M. A., Ratzlaff, C., Doerfling, P., and Kopec, J., "Reliability and validity of an internet-based questionnaire measuring lifetime physical activity," American Journal of Epidemiology, 172 (10), 1190-1198, Nov, 2010.

[44] Hanson, N., Madaras, L., Dicke, J., and Buckworth, J., "Motivational differences between half, full and ultramarathoners," Journal of Sports Behaviour, 38 (2), 180-191, 2015.

[45] Krouse, R. Z., Ransdell, L. B., Lucas, S. M., and Pritchard, M. E., "Motivation, goal orientation, coaching, and training habits of women ultrarunners," Journal of Strength and Conditioning Research, 25 (10), 2835-2842, 2011.

[46] Winters-Hart, C. S., Brach, J. S., Storti, K. L., Trauth, J. M., and Kriska, A. M., "Validity of a questionnaire to assess historical physical activity in older women," Medicine and Science in Sports and Exercise, 36 (12), 2082-2087, Dec, 2004. 